Manuelle Medizin 2015 $53: 410$

DOI 10.1007/s00337-015-0037-z

o Springer-Verlag

Berlin Heidelberg 2015

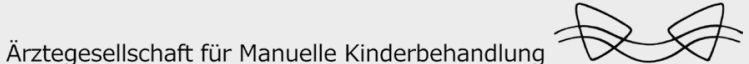

\& Atlastherapie e.V.

\section{Ärztegesellschaft für Manuelle Kinderbehandlung} und Atlastherapie (ÄMKA) e.V.

in Kooperation mit dem Dr. Karl-Sell-Ärzteseminar Neutrauchburg (MWE) e.V.

Anna-von-Borries-Str. 1

D-730625 Hannover

info@aegamk.de

Fax: 0511-5354675

\title{
Kursprogramm 2015
}

\section{Kursprogramm Hannover 2015}

Kursort: Annastift Hannover

Kursleiter: Dr. K. Gilbrich, Dr. R. Kamping, Dr. P. Weng, Dr. M. Ruprecht

Kurs B Diagnostikkurs 2

Atlastherapie nach Arlen Kurs A

18.11.-21.11.2015

Atlastherapie nach Arlen Kurs B

16.09.-19.09.2015

18.11.-21.11.2015

\section{Kursprogramm Hannover 2016}

Kursort: Annastift Hannover

Kursleiter: Dr. K. Gilbrich, Dr. R. Kamping, Dr. P. Weng, Dr. M. Ruprecht

Kinderkurs C: $\quad$ Therapiekurs $1 \quad$ 28.01.-30.01.2016

Kinderkurs D: Therapiekurs 2 21.04.-23.04.2016

Kinderkurs A: $\quad$ Diagnostikkurs 1 14.09.-17.09.2016

Kinderkurs B: $\quad$ Diagnostikkurs 2 16.11.-19.11.2016

Atlastherapie nach Arlen Kurs 1: $\quad$ 14.09.-15.09.2016

Atlastherapie nach Arlen Kurs 2: $\quad$ 16.11.-17.11.2016

\section{Anmeldung ausschließlich über:}

Diakonische Dienste Hannover GmbH

Akademie für Fort- und Weiterbildung

Anna-von-Borries-Str. 1-7, 30625 Hannover

Info-Tel: 0511-5354662, Fax: 0511-5354672

info@ddh.akademie.de, www.ddh-akademie.de

\section{Kursprogramm Isny-Neutrauchburg 2015}

2015 finden in Isny keine Kurse statt, eine neue Kursserie beginnt Anfang des Jahres 2016.

\section{Kursprogramm Isny-Neutrauchburg 2016}

Kurs A

Kurs B

Diagnostikkurs 1

Kurs C

Diagnostikkurs 2

Therapiekurs 1

Kurs D Therapiekurs 2

Atlastherapie Kurs 1

Atlastherapie Kurs 2
27.01.-30.01.2016

16.03.-19.03.2016

15.09.-17.09.2016

24.11.-26.11.2016

27.01.-28.01.2016

16.03.-17.03.2016
Anmeldung:

MWE-Sekretariat

Riedstr. 5, 88316 Isny-Neutrauchburg

Tel: 07562-9718-0, Fax: 07562-9718-22

info@manuelle-mwe.de,www.manuelle-mwe.de

\section{Kinder-Kurs A Diagnostikkurs 1}

Spezielle Radiologie des zervikookzipitalen Überganges (Kopfgelenke Teil 1), praktische Übungen.

Neurophysiologische und manualmedizinische Untersuchung im Säuglingsalter: frühkindliche Entwicklung, Reflexologie, sensomotorische Integration, funktionelle und segmentale Befundung der sensorischen Schlüsselregionen.

\section{Kinder-Kurs B Diagnostikkurs 2}

Kopfgelenk (Teil 2), klinische und physiologische Aspekte, therapeutischer Ansatz, Simulator-Training. Schriftl. Test.

Sensomotorische Dysfunktion im Kindesalter, ADS, Therapiegrundsätze bei idiopathischer Skoliose. Infantile Zerebralparese: Propädeutik und Untersuchungstechnik.

\section{Kinder-Kurs C Therapiekurs 1}

Manuelle Impuls - und Weichteiltechniken bei Säuglingen und Kindern an Wirbelsäule und Extremitäten.

\section{Kinder-Kurs D Therapiekurs 2}

Manuelle Impuls- und Weichteiltechniken an Wirbelsäule und Extremitäten (Teil 2), Atlastherapie bei Säuglingen und Kindern.

Examen. Zertifikat

Für bereits ausgebildete Atlastherapeuten/-therapeutinnen verkürzen sich die Kurse A und B um jeweils 2 Tage

Die Ausbildung in Atlastherapie nach Arlen umfasst in den Kinderkursen A und B die jeweils ersten beiden Tage der Kursdauer. Für bereits ausgebildete Atlastherapeuten/-therapeutinnen verkürzt sich die Kursdauer für die Kinder-Kurse A-B entsprechend.

Voraussetzung für die Kursteilnahme ist die abgeschlossene Weiterbildung in Manueller Medizin. Es werden nur approbierte Ärzte zugelassen. Die Diagnostikkurse sind Voraussetzung für die Teilnahme an den Therapiekursen. 